Nebenwirkungen der Medikation vor allem in Form von erhöhter Gereiztheit und Weinerlichkeit. Auch die Wirkung wurde als mäßig angegeben: Als Ursache wurde eine Studiendesign bedingte zu niedrige Dosierung, aber auch eine schwächere Ansprechbarkeit aufgrund des jungen Alters diskutiert. Von daher ist es eher erstaunlich, dass nach 3 Jahren zunächst noch $65 \%$, weitere 3 Jahre später sogar noch 70,9\% der Kinder unter Medikation war.

Stimulanzien stehen dabei an erster Stelle, was auch ihrer studienbelegten Wirksamkeit entspricht. Die Verordnungshäufigkeit von Atomoxetin, das deutlich schwächer auf die ADHS-Symptomatik wirkt, spielt nur eine geringe Rolle und nimmt im Verlauf noch weiter ab. Auch die Praxis zeigt, dass lediglich eine kleine, möglicherweise genetisch bedingte Subgruppe von Atomoxetin und meist nur in Kombination mit Stimulanzien profitiert. Überraschend hoch ist die Verordnungshäufigkeit von Risperidon, nach 6 Jahren immerhin bei 13,4\% der Studienteilnehmer. Möglicherweise geht eine ADHS, die bereits im Vorschulalter sehr ausgeprägt und therapiebedürftig ist, häufiger mit einer Störung des Sozialverhaltens oder einer AutismusSpektrum-Störung einher, die eine antipsychotische Therapie erforderlich machen.

\title{
Oft vergessen: Impfungen bei Kindern nach Krebserkrankungen
}

\section{Eine intensive Chemotherapie bei Kindern mit Krebserkrankungen führt in aller Regel zu einer lang andauernden Störung der humoralen Immunität. Ein Forscherteam hat nun die Impftiter bei Kindern mit unterschiedlichen Krebs- erkrankungen genauer unter die Lupe genommen.}

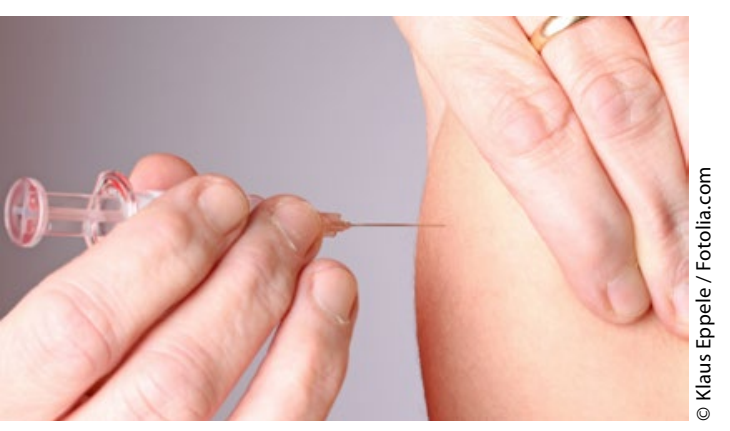

Nach einer Chemotherapie ist die Wiederherstellung des Impfschutzes besonders wichtig.

\footnotetext{
$1 /$ ährend es relativ viele Daten zum Verlust von Impftitern bei Kindern mit akuter lymphatischer Leukämie (ALL) gibt, finden sich zu diesem Thema nur wenig Studien bei Kindern mit Morbus Hodgkin oder bei Kindern mit soliden Tumoren. Aus diesem Grund untersuchte eine Forschergruppe bei Kindern mit verschiedensten malignen Erkrankungen das Risiko, einen vorbestehenden Impfschutz durch eine Chemotherapie zu verlieren.
}

Aufgenommen in die retrospektive Untersuchung wurden Kinder und Jugendliche bis zum Alter von 21 Jahren bei Diagnosestellung einer malignen Grunderkrankung, die zwischen 2001 und 2010 in der Universitätskinderklinik Frankfurt behandelt worden waren. Die Antikörpertiter gegen Masern, Mumps, Röteln und Varizella-ZosterVirus (VZV) wurden zum Zeitpunkt der Diagnosestellung sowie 12 Monate nach Ende der Chemotherapie im gleichen Labor bestimmt. Im Rahmen dieser Studie wurden 195 Kinder (122 Jungen) untersucht. Insgesamt 80 der Patienten litten an einer ALL, 15 an einer akuten myeloischen Leukämie (AML), 18 an einem Non-Hodgkin-Lymphom (NHL), 22 an einem Morbus Hodgkin und 60 an soliden Tumoren unterschiedlicher Art.

Bei der Analyse der Gesamtpopulation zeigte sich, dass insgesamt $27 \%$ der Patienten ihren Impfschutz gegen Masern, $47 \%$ gegen Mumps, $19 \%$ gegen Röteln und $17 \%$ gegen VZV durch die Chemotherapie verloren hatten. Auffällig war, dass der Verlust des Impfschutzes
Die Aussagekraft der Studie wird eingeschränkt durch die hohe Ausfallrate: fast ein Drittel der Patienten - was aber für Langzeitstudien üblich ist. Eine weitere Schwäche ist, dass lediglich das Rating der Eltern berücksichtigt wurde, weder Lehrerurteile noch Bewertungen von betreuenden Ärzten wurden eingesetzt. Es fehlt auch eine Kontrollgruppe zur Beurteilung des Ausmaßes der noch vorhandenen Störungsrelevanz der ADHS-Symptomatik. Dennoch zeigt sich, dass die ADHS-Diagnose konstant ist und Stimulanzien von den meisten Studienteilnehmern weiter als unterstützend empfunden und daher eingenommen werden. Dr. Kirsten Stollhoff vor allem jüngere Kinder betraf. Auch hatten im Vergleich zu Kindern mit AML, NHL, Morbus Hodgkin und soliden Tumoren Kinder mit ALL ein signifikant höheres Risiko, den vorbestehenden Impfschutz zu verlieren, was durch die lange Dauertherapie bei diesen Patienten erklärt werden kann.

Bochennek K et al. Differential loss of humoral immunity against measles, mumps, rubella and varicella-zoster virus in children treated for cancer. Vaccine 2014;32:3357-61

\section{Kommentar}

Die Ergebnisse dieser Studie zeigen deutlich, dass ein signifikanter Anteil der Kinder mit Krebserkrankungen durch die Chemotherapie ihren vorbestehenden Impfschutz verliert. Dies hat einen wichtigen Einfluss auf die Nachbetreuung dieser Kinder. So ist die Wiederherstellung eines durch die Chemotherapie verlorenen Impfschutzes eine wichtige Aufgabe, für die eine enge Zusammenarbeit zwischen betreuenden Hämatologen/Onkologen und niedergelassenen Kinderärzten erforderlich ist. Allerdings ist es bisher noch unklar, wann der optimale Zeitpunkt für die jeweilige Revakzinierung (Effektivität versus Toxizität) ist. Auch ist noch nicht geklärt, inwiefern eine Kontrolle der einzelnen Impftiter vor beziehungsweise nach einer Impfung notwendig ist - was jedoch einen bedeutenden Kostenfaktor darstellt sowie die Lebensqualität der kleinen Patienten beeinflusst (venöse Blutentnahme). Prof. Dr. Thomas Lehrnbecher 